\title{
The quantum cat map on the modular discretization of extremal black hole horizons
}

\author{
Minos Axenides ${ }^{1, \mathrm{a}}$, Emmanuel Floratos ${ }^{1,2, \mathrm{~b}}$, Stam Nicolis ${ }^{3, \mathrm{c}}$ \\ ${ }^{1}$ NCSR "Demokritos", Institute of Nuclear and Particle Physics, 15310 Aghia Paraskevi, Attiki, Greece \\ ${ }^{2}$ Physics Department, University of Athens, Zografou University Campus, 15771 Athens, Greece \\ ${ }^{3}$ CNRS-Institut Denis Poisson (UMR 7013), Université de Tours, Université d'Orléans, Parc Grandmont, 37200 Tours, France
}

Received: 25 October 2017 / Accepted: 26 April 2018 / Published online: 24 May 2018

(C) The Author(s) 2018

\begin{abstract}
Based on our recent work on the discretization of the radial $\mathrm{AdS}_{2}$ geometry of extremal $\mathrm{BH}$ horizons, we present a toy model for the chaotic unitary evolution of infalling single-particle wave packets. We construct explicitly the eigenstates and eigenvalues for the single-particle dynamics for an observer falling into the BH horizon, with as time evolution operator the quantum Arnol'd cat map (QACM). Using these results we investigate the validity of the eigenstate thermalization hypothesis (ETH), as well as that of the fast scrambling time bound (STB). We find that the QACM, while possessing a linear spectrum, has eigenstates, which are random and satisfy the assumptions of the ETH. We also find that the thermalization of infalling wave packets in this particular model is exponentially fast, thereby saturating the STB, under the constraint that the finite dimension of the single-particle Hilbert space takes values in the set of Fibonacci integers.
\end{abstract}

\section{Introduction}

A very interesting revival of the old relation between the near-horizon shock-wave $\mathrm{BH}$ geometries with gravitational memory effects and the information paradox has recently appeared [1-6].

It seems possible in principle that the horizon region of the $\mathrm{BH}$ could form a random basis of purely geometrical data of all of its past and recent history, through the 't Hooft mechanism of permanent space-time displacements caused by high energy scattering events of infalling wave packets [13].

\footnotetext{
a e-mail: axenides@inp.demokritos.gr

b e-mail: mflorato@phys.uoa.gr

c e-mail: stam.nicolis@1mpt.univ-tours.fr
}

In the language of Refs. [4-6], such data can be identified with the soft hair of the $\mathrm{BH}$, whose origin is the infinite number of conservation laws, described by the BMS group.

This new reincarnation of the 't Hooft-Susskind horizon holography provides a new framework to study mechanisms by which past and recent memories of the shock-wave spacetime geometry structure are encoded in the angular and time correlations of the emitted Hawing radiation.

Quite recently, the non-unitarity of the Hawking radiation has been interpreted as a result of integrating the whole BMS soft graviton cloud, accompanying the hard Hawking quanta $[7,8]$.

Although a realistic calculation with a truly chaotic horizon region, in general, still is not possible, some progress could be made using simple mathematical toy models, which can describe how the information carried by infalling wave packets, is scrambled, through a chaotic single-particle Smatrix [9-21].

Proposals for a chaotic, discretized, dynamics for the microscopic degrees of freedom of the stretched horizon have been discussed for quite some time in the literature [22-27].

So it is imperative to separate the issue of the chaotic dynamics and geometry of the near-horizon region from any issues regarding Hawking radiation.

It is possible to do so, when studying probes of extremal black hole horizons; for, in that case, since the Hawking temperature vanishes, while the entropy does not, there is no Hawking radiation.

In this case, it is well known that the near-horizon geometry is described by a metric that factorizes into a radialtemporal part that can be identified as an $\mathrm{AdS}_{2}$ manifold, while the angular part describes the charges.

It is possible to construct a model for random $\mathrm{AdS}_{2}$ geometries, inspired by the shock-wave geometries, necessary for the chaotic dynamics of the probes, by introducing a consistent discretization of the $\mathrm{AdS}_{2}$ near-horizon geometry 
of nearly extremal black holes. This is the so-called modular discretization, $\operatorname{AdS}_{2}[p]$, for every prime integer $p$ [28-31]. In this framework, the entropy of the black hole, is identified with the Kolmogorov-Sinai entropy of the deterministic, chaotic, dynamics of the geometric, microscopic, degrees of freedom, defining the near-horizon geometry.

$\operatorname{AdS}_{2}[p]$ is a specific discrete deformation of its continuous counterpart. It has a random structure due to the modular arithmetic. As explained in Refs. [28-31], by explicit calculation, this specific discretization is chosen among many possible discretizations because it provides a way of constructing an holographic correspondence between the bulk, $\operatorname{AdS}_{2}[p]$ and its boundary $\mathbb{R P}^{1}[p]$, the discrete projective line.

The reason this discrete holography exists at all is that it is possible to realize the action of the discrete and finite symmetry group of $\operatorname{AdS}_{2}[p]$, which is $\operatorname{PSL}_{2}[p]$ : it acts as an isometry group of the bulk and as the (Möbius) conformal group on the boundary.

This discrete geometry provides also a natural framework for describing the single-particle dynamics, via observers, with time evolution operators that are elements of the isometry group. This is a discrete analog of the superconformal quantum mechanics of probes near the horizons of large extremal black holes [32].

In the present work we specify the infalling, accelerating, observer by the well-known Arnol'd cat map (ACM) [3335]. This map defines a particular observer, ACM, who, by performing single-particle scattering on the horizon of the black hole, can probe the randomness of the geometry and it is consistent with the isometries of the background since it belongs to the discrete isometry group of $\operatorname{AdS}_{2}[p]$. The discreteness of the geometry implies that the global coordinates of $\operatorname{AdS}_{2}[p]$ are discrete. On the other hand, the time, measured by the ACM observer, is the iteration step of the corresponding map.

In this work we study the quantum dynamics of the probe in this discrete, background geometry.

It is important to stress that both, probe and background geometry, have a finite dimensional space of states. What we study is how superpositions of the states of the probe evolve, when the background geometry is found in any given fixed state.

The ACM can be "quantized", i.e., it is possible to define a $p \times p$ unitary evolution operator, called the quantum Arnol'd cat map (QACM). This definition uses the Weyl representations of $\mathrm{SL}_{2}[p]$ and especially those that correspond to its projective action, by $\mathrm{PSL}_{2}[p]$ on $\mathrm{AdS}_{2}[p]$. This construction extends the results for the case of the discrete torus [36-43].

An introduction to the requisite tools from arithmetic geometry and computational number theory can be found in Refs. [44-46].

Next we present the plan of the paper.
In Sect. 2 we recall the properties of the ACM, its relation to the Fibonacci sequence and its periods $\bmod p$. We study its group of symmetries, inside $\mathrm{PSL}_{2}[p]$, i.e. the set of elements of $\mathrm{PSL}_{2}[p]$ that commute with it.

In Sect. 3 we construct explicitly the exact quantum Arnol'd cat map, using the metaplectic (or Weyl) representation of $\mathrm{SL}_{2}[p]$, which is reducible and splits into $(p+1) / 2$ and $(p-1) / 2$ dimensional irreducible ones. One of these two unitary irreps, depending on the form of the prime number $p$, is also a representation of $\operatorname{PSL}_{2}[p]$. This particular representation defines, for every prime, $p$, the Hilbert space of states of the infalling wavepackets on $\operatorname{AdS}_{2}[p]$, while the other one is appropriate for the case of the torus.

We determine analytically the spectrum and the eigenstates of the QACM and we compute their degeneracies.

We find the interesting result that, while the spectrum is linear, the eigenstates are chaotic in a very specific way, that is, the squares of the absolute values of the amplitudes (probabilities) are drawn from a (discrete) Gaussian distribution, while their phases have a flat distribution. These results are known to be the premises for the eigenstate thermalization hypothesis (ETH) for quantum ergodicity or unitary thermalization [47-54].

In Sect. 4 we review the Eigenstate Thermalization Hypothesis and we stress that its premises can be checked to hold within our framework. We use the results obtained in Sect. 3 to study the spectrum of scrambling times and we find that for Fibonacci integer values the scrambling time bound of Susskind and Sekino is saturated.

Finally, in Sect. 5 we discuss our results and open problems for future work.

In Appendix A we collect all the necessary material for the detailed construction of the Weyl representation of $\mathrm{SL}_{2}(p)$ and $\operatorname{PSL}_{2}(p)$ and we present the technical details for the analytic construction of the eigenstates and eigenvalues of the QACM.

\section{The Arnol'd cat map and Fibonacci chaos on $\operatorname{AdS}_{2}[N]$}

We review the description of the modular discretization of $\mathrm{AdS}_{2}$ [28-31].

We define the modular discretization by replacing the set of real numbers, $\mathbb{R}$, by the set of integers modulo $N$. The coset finite geometry $\operatorname{AdS}_{2}[N]=S L_{2}\left(\mathbb{Z}_{N}\right) / S O\left(1,1, \mathbb{Z}_{N}\right)$ so obtained is a discrete deformation of its continuous counterpart, $\operatorname{AdS}_{2}[\mathbb{R}]=S L_{2}(\mathbb{R}) / S O(1,1, \mathbb{R}) \cdot \operatorname{AdS}_{2}[N]$ is a finite and random set of points in the embedding Minkowski space-time. When $N$ is prime this is an "arithmetic geometry" in the mathematical literature [44-46], that is, a geometry over a finite field. 

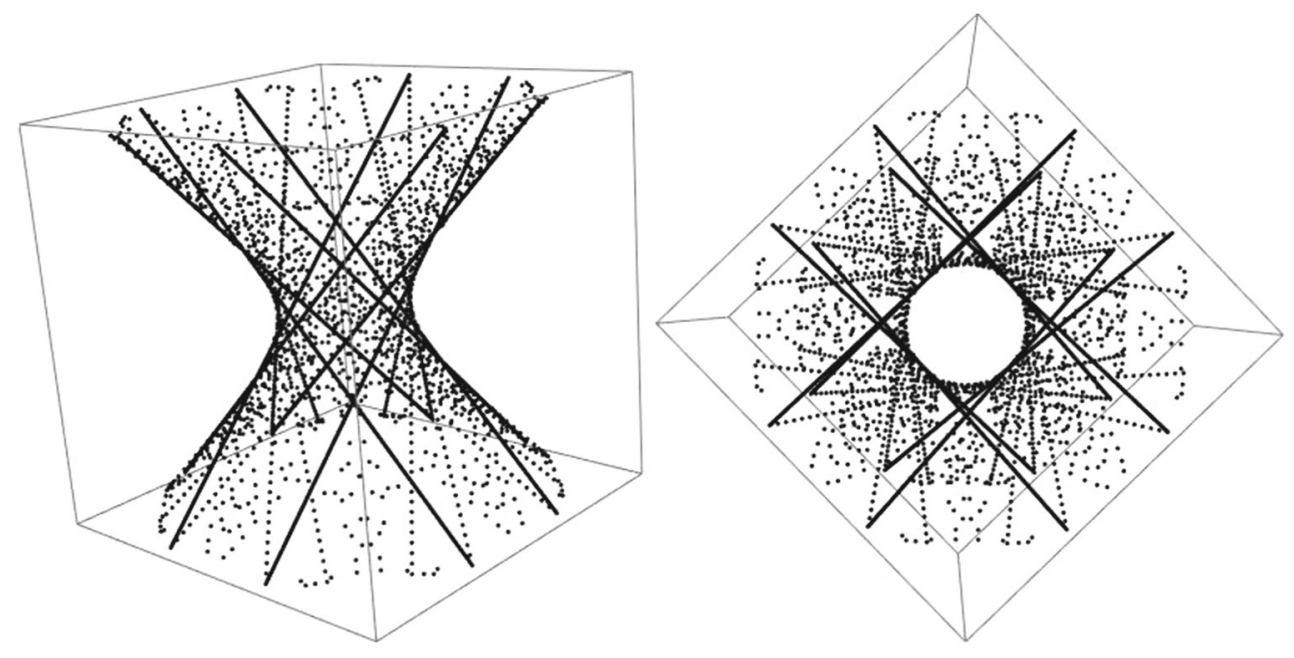

Fig. 1 The rational points on $\operatorname{AdS}_{2}[p]$-side view and top view, for $N=47, M=48, L=144$

This discretization can be used to describe nonlocality, chaos and quantum information processing in the vicinity of the $\mathrm{BH}$ horizon, as well as defining a discrete version of the $\mathrm{AdS}_{2} / \mathrm{CFT}_{1}$ holography.

The set of points of the finite geometry of $\operatorname{AdS}_{2}[N]$ is, by definition, the set of integer solutions mod $N$ of the equation

$x_{0}^{2}+x_{1}^{2}-x_{2}^{2} \equiv 1 \bmod N$

This set-an example of which is shown in Fig. 1is constructed by noting that, for any triplet of integers $(k, l, m)$ that satisfy Eq. (2.1) $\bmod N$, there exists an integer $M \equiv 1 \bmod N$, such that the triple of rational points $(k / M, l / M, m / M) \equiv\left(x_{0}, x_{1}, x_{2}\right)$ satisfies the equation $x_{0}^{2}+x_{1}^{2}-x_{2}^{2}=1$, i.e. it defines rational points of the continuum $\mathrm{AdS}_{2}$ manifold.

If we fix an "infrared cutoff", $L$, for $x_{2},\left|x_{2}\right| \leq L$, by increasing the denominator $M$, we can obtain in this way a rational approximant to the continuum $\mathrm{AdS}_{2}$ geometry.

A straightforward prescription for constructing all the solutions of Eq. (2.1), i.e. the points of $\operatorname{AdS}_{2}[N]$, is as follows:

$x_{0} \equiv(a-b \mu) \bmod N$

$x_{1} \equiv(b+a \mu) \bmod N$

$x_{2} \equiv \mu \bmod N$

where $a^{2}+b^{2} \equiv 1 \bmod N$ and $a, b, \mu \in\{0,1,2, \ldots, N-$ $1\}$ [28-31].

Thus, the discretized, spatial part, along $x_{2}$, consists of $N$ points and the Hilbert space of single-particle states has dimension $N$. The global, $\mathrm{AdS}_{2}$, time, is parametrized by the points of the discrete circle, $a^{2}+b^{2} \equiv 1 \bmod N$. The proper time of the ACM observer is identified with the number of iterations of the $\mathrm{ACM} \bmod N$. Due to the $\bmod N$ arithmetic, the global, $\mathrm{AdS}_{2}$ time and the proper time of the $\mathrm{ACM}$ are periodic.

If we assign to each spatial point a two-state system, the microscopic degrees of freedom of the near-horizon discrete geometry have a Hilbert space of dimension $\propto 2^{N}$. From this we conclude that the entropy, $S_{\mathrm{BH}}$ of such configurations is proportional to $\log 2^{N}=N \log 2$, which is the number of spatial points.

The discussion of the group theoretic properties of this discrete geometry is facilitated, if we restrict $N$ to be a prime integer, $p$. The extension for arbitrary, odd, integer values of $N$ is easily realized by using appropriate factorization theorems [43].

The finite geometry, $\operatorname{AdS}_{2}[p]$, has as isometry group the finite projective modular group, $\mathrm{PSL}_{2}[p]$. This group is obtained as the reduction $\bmod p$, of all elements of $\operatorname{PSL}(2, \mathbb{Z})$. The kernel of this homomorphism is the "principal congruent subgroup", $\Gamma_{p}$. The order of $\operatorname{PSL}_{2}[p]$ is $p\left(p^{2}-1\right) / 2$ and the order of its dilatation subgroup is $(p-1) / 2$; thus, the number of points of $\operatorname{AdS}_{2}[p]$ is $p(p+1)$.

It is easy to find the number of points of $\operatorname{AdS}_{2}[N]$, for any integer $N$.

Numerical experiments suggest the following recursion relation for the number of points of $\operatorname{AdS}_{2}\left[p^{k}\right]$, Sol $\left(p^{k}\right)$ :

$\operatorname{Sol}\left(p^{k}\right)=p^{2(k-1)} \operatorname{Sol}(p) \Rightarrow \operatorname{Sol}\left(p^{k}\right)=p^{2 k-1}(p+1)$

where $\operatorname{Sol}(p)=p(p+1)$ and $k=1,2, \ldots$ for any prime integer $p$.

For $N=2^{n}$ we find $\operatorname{Sol}(2)=4$, $\operatorname{Sol}(4)=24$, and $\operatorname{Sol}\left(2^{k}\right)=4 \operatorname{Sol}\left(2^{k-1}\right)$, for $k \geq 3$. We remark that $N=4$ is an exception. The solution is $\operatorname{Sol}\left(2^{k}\right)=2^{2 k+1}$, for $k \geq 3$. 
Therefore we may deduce the expression for the number of points, for any integer $N$ by prime factorization.

From these results we deduce that, for large $N$, the number of solutions, $\bmod N$, scales like the area, i.e. $N^{2}$. So most of the points of $\operatorname{AdS}_{2}[N]$ are close to its boundary and holography is possible in this case too [55].

Next we discuss the discrete time evolution of the motion of a particle on $\operatorname{AdS}_{2}[N]$. To every point $x_{\mu} \in \operatorname{AdS}_{2}[N]$, where $\mu=0,1,2$, can be assigned a traceless, $2 \times 2$, matrix $X$

$\mathrm{X} \equiv\left(\begin{array}{cc}x_{0} & x_{1}+x_{2} \\ x_{1}-x_{2} & -x_{0}\end{array}\right)$

whose determinant is det $\mathrm{X}=-x_{0}^{2}-x_{1}^{2}+x_{2}^{2} \equiv-1 \bmod N$.

The discrete time evolution for an observer defined by its evolution matrix, $\mathrm{A} \in \mathrm{PSL}_{2}[N]$, is given by the recursion relation

$\mathrm{X}_{n+1}=\mathrm{AX}_{n} \mathrm{~A}^{-1}=\mathrm{A}^{n} \mathrm{X}_{0} \mathrm{~A}^{-n} \bmod N$

where $n=0,1,2, \ldots$ labels the (stroboscopic) time of the observer and $\mathrm{X}_{0}$ is the initial point of the trajectory.

We make a specific choice, introduced in Refs. [28-31], of an observer, described by the Arnol'd cat map (ACM):

$A=\left(\begin{array}{ll}1 & 1 \\ 1 & 2\end{array}\right)$

The map corresponds to successive kicks, forwards and backwards along the light cone of $\mathrm{AdS}_{2}[N]$, since

$A=\left(\begin{array}{ll}1 & 0 \\ 1 & 1\end{array}\right)\left(\begin{array}{ll}1 & 1 \\ 0 & 1\end{array}\right)=L^{-1}$

and it is an element of $\operatorname{PSL}_{2}\left(\mathbb{Z}_{N}\right)$. We point out that this action differs from the action of ACM on the torus, which is linear in A [33-35].

We choose to use this particular map, for the following reasons:

- The ACM has been thoroughly studied for its area preserving action on the classical toroidal phase space and it is known to possess ergodicity, exponentially fast mixing and an infinite number of unstable periodic orbits. The important property of mixing, which is the technical definition of scrambling, assumes that the phase space is compact.

The ACM, acting on $\operatorname{AdS}_{2}[N]$, according to (2.5), induces a discrete Lorentz transformation. The $\bmod N$ prescription guarantees mixing and, thus, ergodicity of the dynamics.
- The $\bmod N$ prescription provides chaotic orbits on $\operatorname{AdS}_{2}[N]$ but, since ACM has a finite period, $T(N)$, depending, randomly, on $N$, all of these orbits are, also, periodic. Their chaotic nature can be seen up to evolution time $\leq T(N) / 2$.

- The ACM has been studied intensively also as a toy model for semiclassical quantum chaos on the toroidal phase space [36-39], although the degeneracies in its spectrum impose additional constraints on its quantum ergodic properties [56-60].

Here we extend the study to the classical and quantum motion of particles under the Arnol'd cat map on the discretized $\operatorname{AdS}_{2}[N]$ geometry.

As we pointed out above, this is a discretized deformation of the continuous, radial and time, geometry of the near-horizon region of extremal BHs [61-63].

The motion we study is the longitudinal motion of probes and it differs from the motion along the horizon of the black hole, which is the two dimensional sphere. On the other hand, physically the scrambling of information on the horizon happens at the same time as the longitudinal (radial) scrambling [64,65].

An important property of ACM is that it is well known to generate the sequence of Fibonacci numbers, $f_{n}$, with $n=$ $1,2, \ldots$, defined by

$\left(\begin{array}{c}f_{n} \\ f_{n+1}\end{array}\right)=\left(\begin{array}{ll}0 & 1 \\ 1 & 1\end{array}\right)\left(\begin{array}{c}f_{n-1} \\ f_{n}\end{array}\right)$

where $f_{0}=0$ and $f_{1}=1$.

We observe that

$A=\left(\begin{array}{ll}1 & 1 \\ 1 & 2\end{array}\right)=\left(\begin{array}{ll}0 & 1 \\ 1 & 1\end{array}\right)^{2}$

therefore

$\mathrm{A}^{n}=\left(\begin{array}{cc}f_{2 n-1} & f_{2 n} \\ f_{2 n} & f_{2 n+1}\end{array}\right)$

with $n=1,2,3, \ldots$

Falk and Dyson [66] studied the periods, $T(N)$, of the iteration

$\mathrm{A}^{n} \bmod N=\left(\begin{array}{cc}f_{2 n-1} & f_{2 n} \\ f_{2 n} & f_{2 n+1}\end{array}\right) \bmod N$

for various classes of the integers $N$.

$T(N)$ is the smallest, positive, integer, such that

$\mathrm{A}^{T(N)}=\left(\begin{array}{ll}1 & 0 \\ 0 & 1\end{array}\right) \bmod N$ 


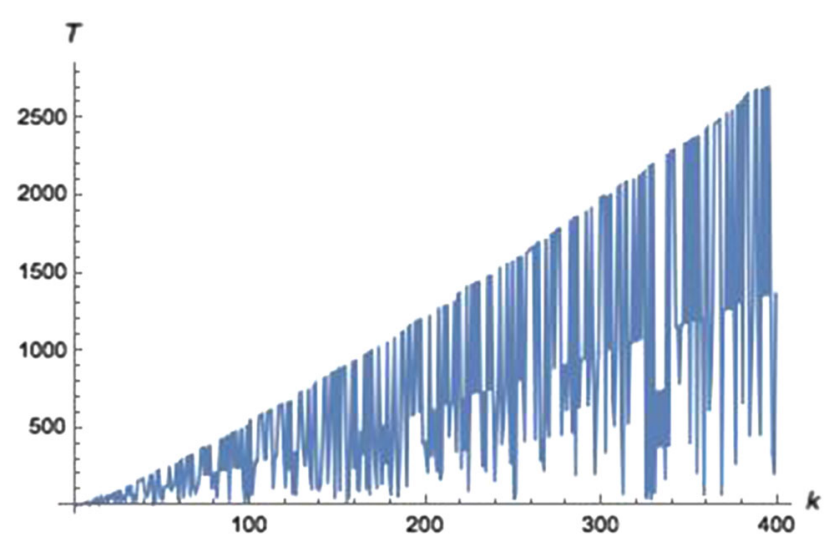

Fig. 2 The period, $T$, of the Arnol'd cat map as a function of the order, $k$, of the prime $p_{k}$, for the first 400 primes

Thus $T(N)$ is, also, the period of the Fibonacci sequence $\bmod N$, which is well known to be a "random" function of $N$; cf. Fig. 2.

We now turn to the discussion of the scrambling time, $t_{\text {scrambling, }}$, of the black hole horizon geometry, which has been introduced in [67-72] as the time necessary for uniform spreading of the distribution of the microscopic degrees of freedom, near the black hole horizon, under an external perturbation. Here we use the probe approximation for an infalling, single-particle, wavepacket as such a perturbation and we assume that the scrambling time of the black hole horizon is the same as that of the wavepacket. For a Gaussian wavepacket the scrambling time is the time necessary for its uniform spreading along the horizon. Moreover, we focus on the radial dependence of the spreading, for which the scrambling time is the same as that of the transverse spreading.

More technically, the scrambling time, $t_{\text {scrambling, as }}$ defined above, is identical to the mixing time, $t_{\text {mixing }}$ of the dynamical system, here the cat map, on $\operatorname{AdS}_{2}[N]$ [33-35]. Since $T(N)$ is the period of $A \bmod N$, the maximum available time for scrambling is proportional to $T(N) / 2$, therefore

$t_{\text {scrambling }}=t_{\text {mixing }} \leq \frac{T(N)}{2}$

for all $N$.

From the work of Falk and Dyson [66], if $N=f_{2 k}$, then $T(N)=2 k$. Therefore, $t_{\text {scrambling }}=t_{\text {mixing }} \leq k$. We recall that the solution of the Fibonacci recurrence is given by

$f_{n}=\frac{1}{\sqrt{5}}\left(\lambda^{n}-\frac{(-)^{n}}{\lambda^{n}}\right)$

with

$\lambda=\frac{1+\sqrt{5}}{2}$, known as the Golden Ratio. For $n=2 k>>1, f_{2 k}=N \sim$ $(1 / \sqrt{5}) \exp (2 k \log \lambda)$, so $T(N) \sim \ln N$. These orbits $\bmod$ $f_{2 k}$ are "short" orbits and, in order to get mixing, we have to take "large" values of $k$. At the quantum level, the role of "short" orbits has been connected with that of "scars" [56$60]$.

Such "short" periods of the ACM imply the existence of non-trivial conservation laws, that is, elements of $S L\left(2, \mathbb{Z}_{N}\right)$ that commute with it. These form an abelian group, the commutant, $G(\mathrm{~A})$. For prime values of $N$, it is cyclic, i.e. there exists a "primitive element", whose powers generate all the others. Among the elements of this group, obviously, are the powers of $\mathrm{A} \bmod N$; the non-trivial conservation laws are described by the complement thereof. The general element of $G(\mathrm{~A})$ has the form

$\mathrm{C}(k, l)=\left(\begin{array}{ll}k & l \\ l & k+l\end{array}\right)$

with $k, l$ integers, satisfying the constraint $k^{2}+k l-l^{2}=1$. This can be cast in the form of Pell's equation,

$x^{2}-5 y^{2}=1$

with $x=k+(l / 2)$ and $y=l / 2$, in which case $l$ must be even. The "trivial" conservation laws are given by the Fibonacci numbers, $k=f_{2 n-1}, l=f_{2 n}$, for all $n$; in this case, integer solutions of Pell's equation correspond to $n=3 m$, with $m=1,2, \ldots[44-46,73]$.

For prime values of $N$ the period of the ACM divides the period of the commutant. If the two periods are equal, the $\mathrm{ACM}$ is a primitive element of $G(\mathrm{~A})$ and there are no non-trivial conservation laws at all. If they are not equal, then the ACM is a power of the primitive element of the commutant. This power determines the degeneracies of the quantum $\mathrm{ACM}$, as we shall see in the next section.

The deterministic, chaotic, orbits of ACM on $\mathrm{AdS}_{2}[N]$ can be obtained as follows: If we take as the initial point $\mathrm{X}_{0} \equiv\left(x_{0}, x_{1}, x_{2}\right)$, we find the corresponding sequence, $\left\{\mathrm{X}_{n}\right\}$,

$$
\begin{aligned}
& \mathrm{X}_{n} \equiv\left(\begin{array}{cc}
x_{0}^{(n)} & x_{1}^{(n)}+x_{2}^{(n)} \\
x_{1}^{(n)}-x_{2}^{(n)} & -x_{0}^{(n)}
\end{array}\right)=\mathrm{A}^{n} \mathrm{X}_{0}\left[\mathrm{~A}^{-1}\right]^{n} \\
& =\left(\begin{array}{cc}
f_{2 n-1} & f_{2 n} \\
f_{2 n} & f_{2 n+1}
\end{array}\right)\left(\begin{array}{cc}
x_{0} & x_{1}+x_{2} \\
x_{1}-x_{2} & -x_{0}
\end{array}\right) \\
& \left(\begin{array}{cc}
f_{2 n+1} & -f_{2 n} \\
-f_{2 n} & f_{2 n-1}
\end{array}\right) \bmod N .
\end{aligned}
$$

Writing out the results we find that $\left(x_{0}^{(n)}, x_{1}^{(n)}, x_{2}^{(n)}\right)$ is given by the action of elements $\mathrm{L}_{n} \in S O(2,1)$, with integer coefficients $\bmod N$, that act on the initial point $\left(x_{0}, x_{1}, x_{2}\right)$, for every time step $n$ : 
$\mathrm{L}_{n} \equiv\left(\begin{array}{ccc}\left(f_{2 n}\right)^{2}+f_{2 n-1} f_{2 n+1} & f_{2 n} f_{2 n+1}-f_{2 n} f_{2 n-1} & -f_{2 n} f_{2 n-1}-f_{2 n} f_{2 n+1} \\ f_{2 n} f_{2 n+1}-f_{2 n} f_{2 n-1} & -\left(f_{2 n}\right)^{2}+\frac{1}{2}\left(f_{2 n-1}\right)^{2}+\frac{1}{2}\left(f_{2 n+1}\right)^{2} & \frac{1}{2}\left(f_{2 n-1}\right)^{2}-\frac{1}{2}\left(f_{2 n+1}\right)^{2} \\ -f_{2 n} f_{2 n-1}-f_{2 n} f_{2 n+1} & \frac{1}{2}\left(f_{2 n-1}\right)^{2}-\frac{1}{2}\left(f_{2 n+1}\right)^{2} & \left(f_{2 n}\right)^{2}+\frac{1}{2}\left(f_{2 n-1}\right)^{2}+\frac{1}{2}\left(f_{2 n+1}\right)^{2}\end{array}\right)$.

It is noteworthy that $L_{n}$ has fractional coefficients, which means, even without applying the mod operation, that the corresponding combinations of integer coordinates on the hyperboloid are even. Also we note that, for even $N$, these matrices must be defined separately.

The relevance of these expressions is that they highlight the, classical, deterministic, chaotic dynamics of the ACM on $\operatorname{AdS}_{2}[N]$.

Indeed, the motion of a particle under ACM, along the spatial direction, $x_{2}$, of $\operatorname{AdS}_{2}[N]$ can be seen to be fully chaotic and mixing; cf. Fig. 3.

\section{Chaotic eigenstates of the QACM on $\mathbf{A d S}_{2}$}

Quantum mechanics for a local observer in $\operatorname{AdS}_{2}$ is defined once a choice of time evolution has been made. As discussed in the previous section the isometry group $S L(2, \mathbb{R})$, can be used to identify the time evolution operator for an observer with an element of this group.

Since we have discretized the geometry locally, the canonical variables, for any observer, are the exponentials of position and momentum operators that define the generators of the finite Heisenberg-Weyl group, $\mathrm{HW}_{N}$.

The classical isometry of the discretized geometry is $\operatorname{PSL}\left(2, \mathbb{Z}_{N}\right)$. Having chosen as time evolution map, A, the Arnol'd cat map, we have, in fact, specified the observer, and its time is defined as the number of iterations of this map. We can now construct the corresponding quantum evolution map, QACM, choosing, for simplicity, $N=p$ prime. This will be, also, the dimension of the single-particle Hilbert space of this observer.

The unique Hilbert space, for all observers, is defined by the irrep of the Heisenberg-Weyl group, as we discussed in Refs. [28-31]. Every observer in the bulk can reconstruct the algebra of his/her observables from those of the conformal field theory (for $\mathrm{AdS}_{2}$, this is conformal quantum mechanics) on the boundary using bulk-to-boundary Green functions.

To construct the unitary (quantum) evolution operator, $U(\mathrm{~A})$, corresponding to the classical Arnol'd cat map, A, we shall use the Weyl representation of $\mathrm{PSL}_{2}[p]$.

The detailed construction of $U(\mathrm{~A})$ is given, for completeness, in Appendix A, for the group $\mathrm{SL}_{2}[p]$. This representation, by construction, is the direct sum of two, irreducible, representations, of dimensions $(p+1) / 2$ and $(p-1) / 2$. Since the action of $A$ on $\mathrm{AdS}_{2}$ does not distinguish the action of $A$ from that of $-A$, it realizes a projective action-which is how quantum mechanics on $\mathrm{AdS}_{2}$ differs from the torus, $\mathbb{T}^{2}$.
Therefore it is necessary to choose one of these two representations, thereby imposing the constraint that it is, also, a representation of $\mathrm{PSL}_{2}[p]$.

One important property of the quantization procedure is that, for any two elements, $\mathrm{A}_{1}, \mathrm{~A}_{2} \in \mathrm{PSL}_{2}[p], U\left(\mathrm{~A}_{1} \mathrm{~A}_{2}\right)=$ $U\left(\mathrm{~A}_{1}\right) U\left(\mathbf{A}_{2}\right)$. This implies the interesting fact that, to calculate the quantum evolution, at time $n=1,2,3, \ldots$, it suffices to compute $U\left(\mathrm{~A}^{n}\right)$, which is equal to $[U(\mathrm{~A})]^{n}$-realizing a very big simplification in the calculation of time correlation functions. Therefore, the period of the quantum map is equal to that of the classical map and this determines the degeneracies of the spectra and the conservation laws.

In the following we sketch the main steps of the construction of the eigenstates and eigenvalues of the QACM.

We remark that the restriction to prime values of $N$ for which 5 is a quadratic residue mod $N$ will make possible the analytic construction of eigenstates and eigenvalues of the QACM. It appears that, up to now, explicit expressions for the eigenstates and eigenvalues of the QACM are not known for generic $N$ [36-39]; so our results, for these primes, are new.

The basic idea comes from the observation that the classical ACM can be diagonalized over the finite field $\mathbb{F}_{p}=$ $\{0,1,2, \ldots, p-1\}$, if 5 is a quadratic residue $\bmod p$. So to avoid unnecessary technical complications, we choose the prime $p$ to be of the form $4 k-1$, since, in that case, if 5 is a quadratic residue $\bmod p$ it is easy to construct $\sqrt{5} \bmod p$. (If 5 is not a quadratic residue mod $p$, we must work in the corresponding quadratic extension.)

We can check, in this case, that $a \equiv 5^{k} \bmod p$ satisfies $a^{2} \equiv 5 \bmod p$. The eigenvalues of $\mathrm{A}$ are then

$\lambda_{ \pm} \equiv \frac{3 \pm a}{2} \bmod p$.

Moreover, there is an element, $\mathrm{R} \in \mathrm{SL}_{2}[p]$, that diagonalizes A,

$A=R D_{A} R^{-1}$

where $D_{A}=\operatorname{diag}\left(\lambda_{+}, \lambda_{-}\right)$. We can deduce that

$U(\mathrm{~A})=U(\mathrm{R}) U\left(\mathrm{D}_{\mathrm{A}}\right) U(\mathrm{R})^{\dagger}$

and that $U\left(\mathrm{D}_{\mathrm{A}}\right)$ is the circulant matrix

$\left\langle l\left|U\left(\mathrm{D}_{\mathrm{A}}\right)\right| k\right\rangle=\delta_{\lambda_{+} k, l}=\delta_{k, \lambda_{-} l} \quad k, l=0,1, \ldots, p-1$. 


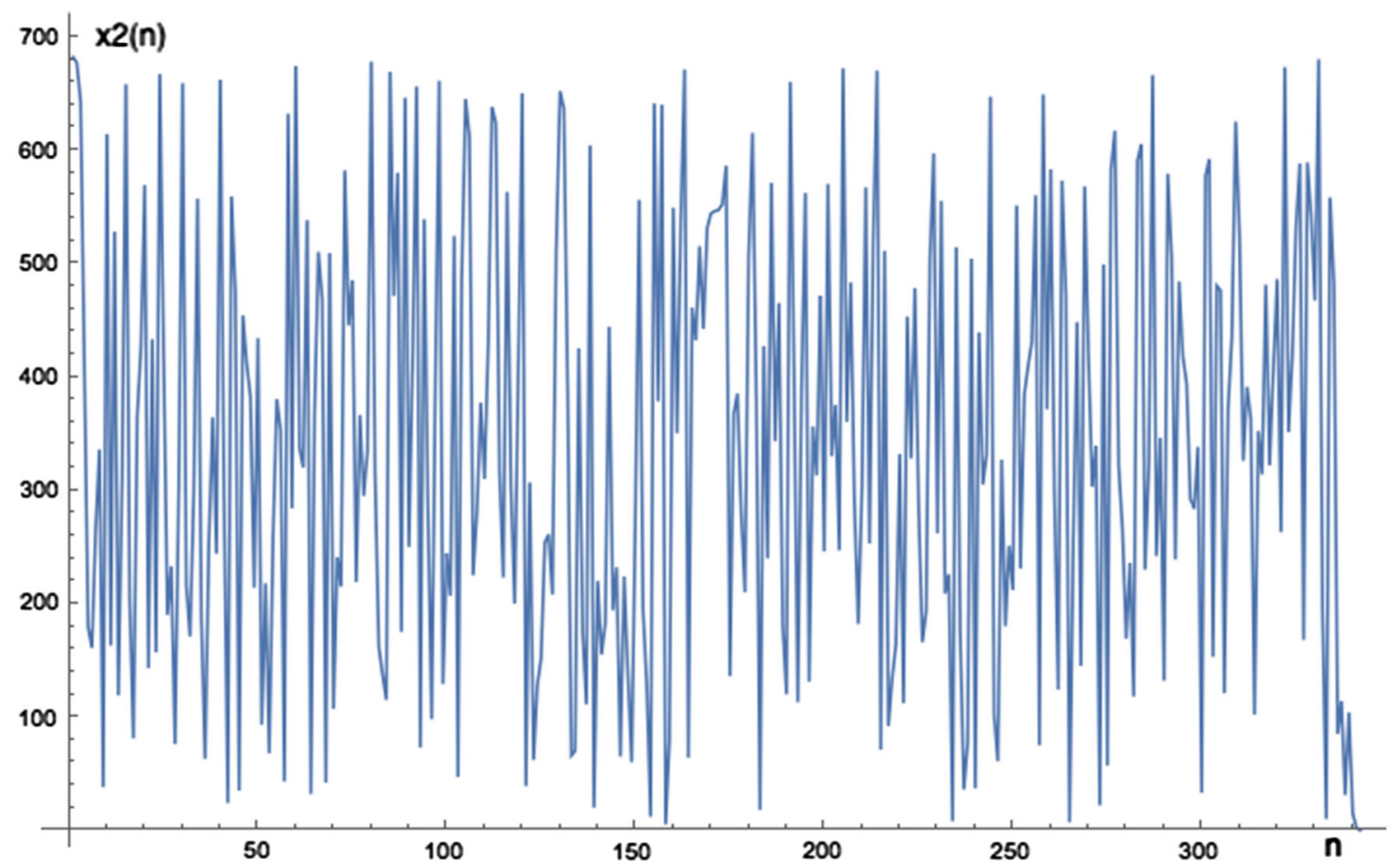

Fig. $3 x_{2}^{(n)} \bmod 683$ as a function of the time step $n$. The period is found to be equal to $684=N+1$

The eigenstates of $U\left(\mathrm{D}_{\mathrm{A}}\right)$ are the multiplicative characters, $\left|\pi_{0}\right\rangle,\left|\pi_{1}\right\rangle, \ldots,\left|\pi_{p-1}\right\rangle$, of $\mathbb{F}_{p}^{*}=\{1,2, \ldots, p-1\}$, given by the expressions

$$
\begin{aligned}
\left\langle k \mid \pi_{0}\right\rangle & =\delta_{k, 0} \quad k=0,1,2, \ldots, p-1, \\
\left\langle 0 \mid \pi_{n}\right\rangle & =0, \\
\left\langle k \mid \pi_{n}\right\rangle & =\frac{e^{\frac{2 \pi i n}{p-1} \operatorname{Ind}_{g}(k)}}{\sqrt{p-1}},
\end{aligned}
$$

where $k, n=1,2, \ldots, p-1$ and $\operatorname{Ind}_{g}(k)$ is the discrete logarithm of $k$ with respect to the base $g$, where $g$ is a primitive element of $\mathbb{F}_{p}^{*}$; i.e.

$$
g^{\operatorname{Ind}_{g}(k)} \equiv k \bmod p
$$

It follows that $\operatorname{Ind}_{g}(k \cdot l)=\operatorname{Ind}_{g}(k)+\operatorname{Ind}_{g}(l)$.

Having determined the eigenstates of $U\left(\mathrm{D}_{\mathrm{A}}\right)$, let us now provide the expressions of the eigenvalues. We remark that

$$
\begin{aligned}
& U\left(\mathrm{D}_{\mathrm{A}}\right)\left|\pi_{0}\right\rangle=\left|\pi_{0}\right\rangle \\
& U\left(\mathrm{D}_{\mathrm{A}}\right)\left|\pi_{n}\right\rangle=e^{-\frac{2 \pi \mathrm{i}}{p-1} n \operatorname{Ind}_{g}\left(\lambda_{+}\right)}\left|\pi_{n}\right\rangle
\end{aligned}
$$

and it can be read off the eigenvalues of $U(\mathrm{~A})$. The eigenvectors of $U(\mathrm{~A}),\left|\psi_{n}\right\rangle$, are given by

$\left|\psi_{n}\right\rangle=U(\mathrm{R})\left|\pi_{n}\right\rangle$.
This calculation becomes effective using the explicit form of $U(\mathrm{R})$, derived from the Weyl representation (cf. Appendix A).

The period of QACM is the period, $T(p)$, of the ACM; and it is also the order of the element(s), $\lambda_{ \pm}$. Since the $\lambda_{ \pm}$ are integers in $\mathbb{F}_{p}^{*}$, this order divides $p-1$, the order of $\mathbb{F}_{p}^{*}$. So there exists an integer, $\tau_{p}$, such that $p-1=\tau_{p} T(p)$. Therefore, $\operatorname{Ind}_{g}\left(\lambda_{+}\right)=\tau_{p}$.

This is, precisely, the degeneracy of the eigenvalues of QACM, which are phases, $e^{\mathrm{i} \varepsilon_{n}}$. From the above we obtain

$\varepsilon_{n}=\frac{2 \pi}{p-1} \tau_{p} n$

with $n=0,1,2, \ldots, p-1$. Since $n$ labels the eigenstates too and $\tau_{p} /(p-1)=1 / T(p)$, we can determine the degenerate eigenstates.

With these tools we can write explicit expressions for the eigenstates, $\left|\psi_{n}\right\rangle$,

$$
\begin{aligned}
\left\langle k \mid \psi_{n}\right\rangle & =\sum_{l=0}^{p-1}\langle k|U(\mathrm{R})| l\rangle\left\langle l \mid \pi_{n}\right\rangle \\
& \Rightarrow\left\{\begin{array}{l}
\left\langle k \mid \psi_{0}\right\rangle=\langle k|U(\mathrm{R})| 0\rangle \\
\left\langle k \mid \psi_{n}\right\rangle=\frac{1}{\sqrt{p-1}} \sum_{l=1}^{p-1}\langle k|U(\mathrm{R})| l\rangle e^{\frac{2 \pi \mathrm{i}}{p-1} n \tau_{p}}
\end{array}\right.
\end{aligned}
$$

where $k=0,1,2, \ldots, p-1$, which will help to understand their chaotic properties. 
The degeneracies of the spectrum imply the existence of non-trivial conservation laws that reduce the size of the attractor. As discussed in the last part of Sect. 2, we can determine explicitly, depending on $p$, the commutant of $A$, $G(\mathrm{~A})$.

This group is cyclic since we have chosen prime values for $p$, and if its order is different from the period of ACM there is a unique element, $\mathrm{B}$, which generates $G(\mathrm{~A})$. The corresponding quantum operator, $U(\mathrm{~B})$, generates the quantum conservation laws.

\section{ETH and the scrambling time bound for the QACM}

Recently, there has been a lot of activity around the question of the thermodynamics of closed quantum systems [47-54].

An important role in this question has been assigned to the specific mechanisms of thermalization of various subsystems.

A particularly interesting proposal is the Eigenstate Thermalization Hypothesis (ETH) [47-54]: The time average of any observable of a subsystem of a closed quantum system which is, initially, in a pure state for large times converges to the thermal average of the observable, along with exponentially small corrections, $O\left(e^{-S}\right)$, where $S$ is the entropy, defined by the thermal density matrix of the system.

Since the total system is closed, it can be taken to be in a pure state and the temperature in the thermal density matrix is an effective temperature, defined by the energy average in the initial state of the total system.

It has been shown [47-54] that a way to realize this hypothesis is to assume that the closed quantum system has a complete set of chaotic states in the specific sense that their probabilities are sampled from a Gaussian pdf, while their phases are sampled from a flat pdf.

These are the basic premises for thermalization to be possible.

In our particular chaotic, quantum, model of singleparticle scattering in the near-horizon region of an extremal black hole, (some of) the basic ingredients of the ETH scenario can be unambiguously identified.

As we shall show below, the system of the black hole near-horizon geometry, including the infalling wavepacket as a subsystem, has a complete set of chaotic eigenstates with, precisely, these properties.

An important quantity that describes thermalization is the time required for thermalization. Recent studies have highlighted the relevance of the Hamiltonian dynamics of integrable and chaotic systems for determining the thermalization time.

On the other hand, for unitary thermalization of the subsystem, it is interesting to study how—and if-the time required is bounded from below as a function of the entropy, for various physical systems [67-72].

It has been conjectured that black hole horizons, considered, along with their probes, as closed quantum systems, are the fastest scramblers [67-72].

$\mathrm{ETH}$, therefore, is a very interesting framework within which to discuss these issues.

It has recently been conjectured that such a bound exists and that it is proportional to $(\beta / 2 \pi) \log S$, with $S$ the entropy and $\beta$ the (inverse) temperature and that black holes saturate it.

In the AdS/CFT approach to this problem, we have the tools to study thermodynamics of gravitational backgrounds through the thermodynamics of the boundary, nongravitational, conformal field theory. This is a consistent description of the thermodynamics of local, gravitational, observers, for which the observables are, indeed, defined unambiguously on the boundary. These are the sources for the boundary conformal field theory.

Chaos is realized within the ETH, assuming that the dynamics of the closed quantum system is ergodic and mixing. This can be shown, using a random matrix description for the dynamics [47-54]. For the case of the black hole, chaos can be described by shock-wave geometries in the near-horizon region. For the thermodynamics of the black hole we expect to obtain a random ensemble of geometries and to determine its measure. Assuming ergodicity and a unitary, gravitational, dynamics, this is equivalent to picking out a "typical" random geometry, as a gravitational background.

Our approach to this end is to use a particular arithmetic, namely a modular discretization of the geometry, which, while random, is consistent with unitarity and holography.

In the following we shall present arguments that support the statement that the QACM eigenfunctions do satisfy the assumptions of the ETH.

The chaotic properties of the eigenstates can be traced back to the chaotic character of the discrete logarithm, $\operatorname{Ind}_{g}(l)$. The definition of chaos we shall adopt, which is the only one consistent with computational and algorithmic complexity, is that of algorithmic chaos.

The effective computation of the discrete logarithm is a classic example of a non-compressible algorithm, i.e. that cannot be done in polynomial time, with respect to the number of the input bits of $l[44-46,73]$. On the other hand, using quantum algorithms, Shor and others have shown that it can be reduced to polynomial complexity [74]

These considerations are consistent with the results of an old but very interesting paper of Ford et al. in Refs. [36-39], which shows that the complexity of the QACM is $(\log N)^{2}$, in contrast with the classical one, which is $N$. This paper created a lot of discussion in the quantum chaos community (cf. the paper by Berry in Les Houches 1989 [36-39]). 


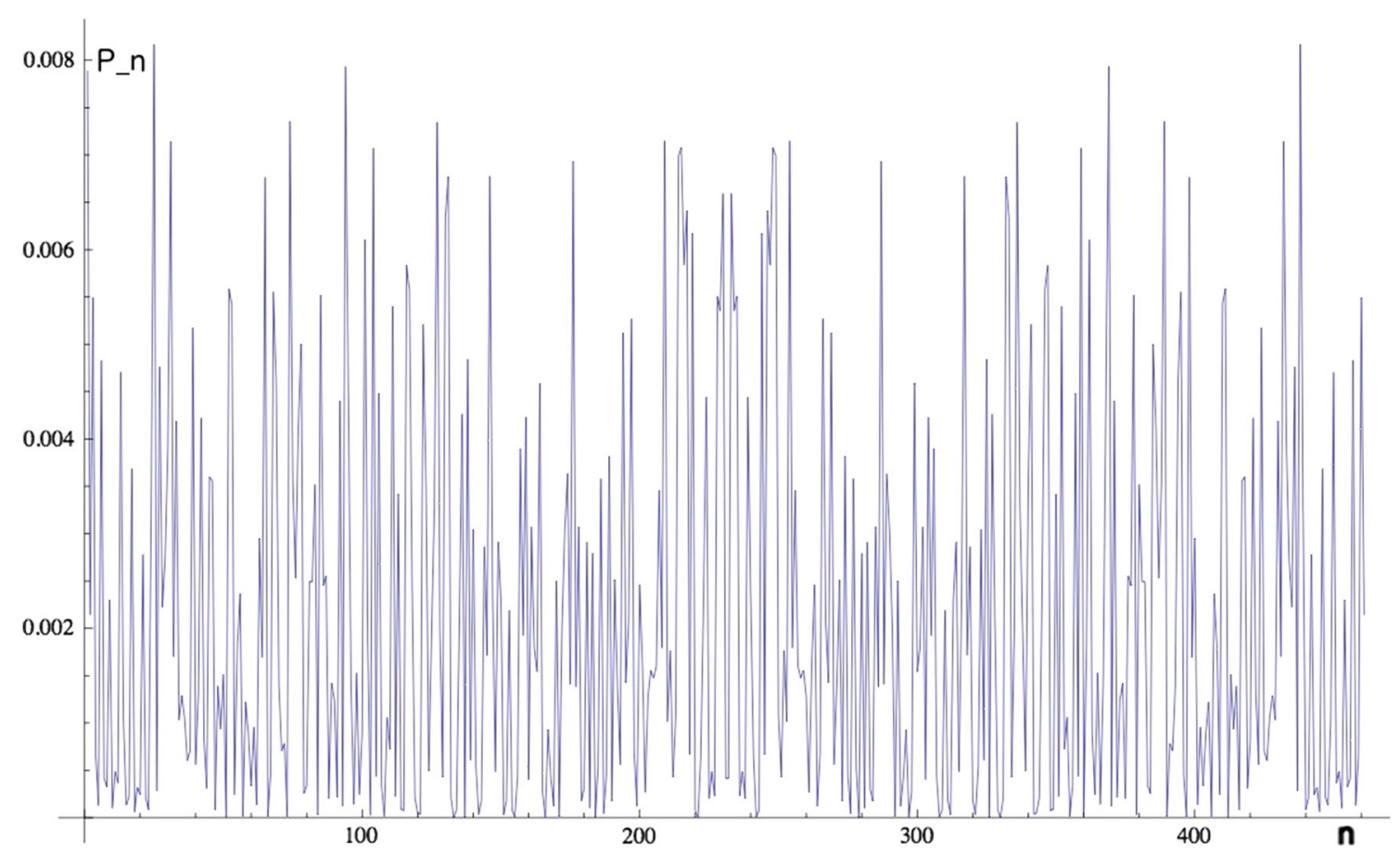

Fig. 4 The squared amplitude, $P_{n} \equiv\left|a_{n}\right|^{2}$, vs. the component label, $0 \leq n \leq p-1$, in the symmetric ground state (in the subspace of dimensionality $(p-1) / 2)$ of the quantum Arnol'd cat map, for $p=461$

The explicit expressions for the states $\left|\psi_{n}\right\rangle$ are sums of random phases, with fixed, complex, amplitudes. This leads, using the large number theorems, for $p \rightarrow \infty$, to Gaussian distributions of the state components. In the next section, we will provide numerical evidence for this claim and shall discuss some of the consequences regarding the randomness of the matrix QACM itself.

As discussed in Sect. 2 the mixing time for the classical ACM scales as the logarithm of the discretization parameter $N$ whenever $N$ takes values in the Fibonacci sequence. The time required for unitary thermalization of a wavepacket (the scrambling time) is identified here with the mixing time $\left(t_{\text {scrambling }}=t_{\text {mixing }}\right)$. This is so because the period of the classical and the quantum ACMs coincide as a result of the construction of $U(A)$.

We find thus that the scrambling time of the QACM is proportional to $\log N$ when the dimension of the singleparticle Hilbert space, $N$, takes values in the sequence of Fibonacci integers. We recall also that the entropy $S$ of the $\mathrm{AdS}_{2}$ is proportional to $N$, the spatial extent of the geometry. This leads to the saturation of the scrambling time bound, $t_{\text {mixing }}=t_{\text {scrambling }} \leq \log S$, of Hayden-Preskill and Sekino-Susskind.

The prefactor, which would be the inverse of the temperature, here is to be replaced by the effective temperature of the closed quantum system, which depends on the complete set of its chaotic eigenstates, according to the ETH scenario. For, while the Hawking temperature of the extremal black hole is zero, the chaotic dynamics of the extremal black hole microstates defines a consistently closed system, since the extremal black hole does not radiate. This point deserves a fuller analysis, which will be reported in future work.

We may define finally the single-particle scattering Smatrix as the evolution operator, evaluated at half the period of the QACM.

A consequence of the chaotic character of the QACM eigenstates is that this matrix is random and completely delocalizes and scrambles initial Gaussian wavepackets.

Closing this section we shall present numerical support of our arguments for the chaotic nature of the eigenstates of QACM (cf. Fig. 4 for the ground state for $p=461$ ). These results were obtained by using Mathematica codes. For $p=461,5$ is a quadratic residue; but 461 is not of the form $4 k-1$; however, it is possible to obtain the eigenvalues and eigenstates of the QACM numerically. In addition, for $p=$ 461, there exist two invariant subspaces, of dimensionalities $(p+1) / 2=231$ and $(p-1) / 2=230$. The projective representation, which is appropriate for $\mathrm{AdS}_{2}$, as discussed above, is the second one. To highlight the symmetry of the ground state, we have not projected onto the half length, but we display the full length.

From this figure it is possible to deduce that the probability distribution function (PDF) for the values of the amplitude squared is, indeed, Gaussian, and the phases are uniformly distributed; cf. Fig. 5. 

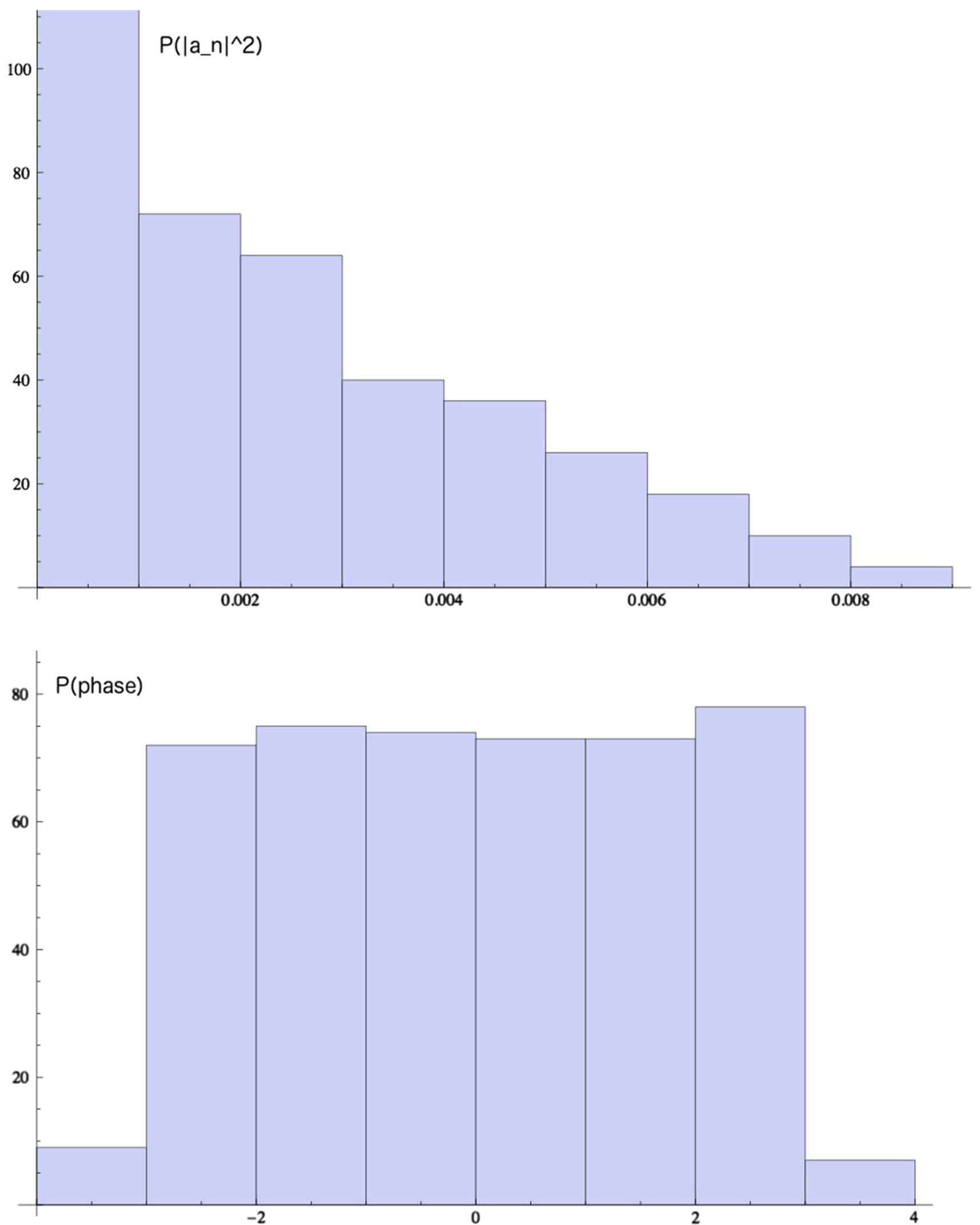

Fig. 5 The binned histograms for the squared amplitude, $\left|a_{n}\right|^{2}$, and for the (relative) phase of the amplitude in the ground state, for $p=461$ 
For Fibonacci dimensions of the Hilbert space of states, the period grows as $\log p$, which saturates the STB. So, for large $p$, an exponentially small part of space-time contributes to the chaotic dynamics; the rest are copies of it. This means that there is a large number of "islands of chaos". ETH thus holds within each such island separately.

This holds for the dynamics of the probes of the radial and temporal, $\mathrm{AdS}_{2}$, geometry of the near-horizon region; far from the horizon, at distances large compared to $\log p$, in units of the $\mathrm{AdS}_{2}$ radius, the behavior becomes regular. The detailed crossover remains to be elucidated.

Of course, this numerical analysis is suggestive and will be completed in future work; but the big picture it defines is expected to be valid.

\section{Summary and conclusions}

In this work we proposed a toy model for the chaotic scattering of single-particle wave packets in the modular discretization of the radial $\mathrm{AdS}_{2}$ space-time geometry of extremal (or nearly) extremal BHs. In recent discussions of the chaotic scattering the focus has been on the dynamics of the microscopic degrees of freedom on the stretched horizon. It is evident that, although the dynamics of longitudinal and transverse scrambling will be different, the time duration will be the same $[64,65]$.

We were able to discretize the coset structure of the geometry of this space-time, by introducing a modular invariant, infrared and ultraviolet cutoff. We obtained an $\mathrm{AdS}_{2} / \mathrm{CFT}_{1}$ holography and we provided the eigenstates and eigenvalues for the quantum chaotic Arnol'd cat map, as well as the single-particle S-matrix.

These eigenstates are chaotic in the sense of the eigenstate thermalization hypothesis. They create mixing and chaos for any infalling Gaussian wave packet. An interesting property of this model is that we can fix the dimension of the singleparticle Hilbert space of states, so as to saturate the scrambling time bound of Hayden-Preskill, Sekino and Susskind, for an observer with time evolution defined by the QACM.

Our results provide a toy model mechanism to explain how the incoming information of a pure state can be scattered back as thermal radiation described by a density matrix (through the ETH scenario), while at the same time preserving unitarity in the single-particle Hilbert space.

For future research along these lines, we think it would be interesting to extend this toy model to the construction of the many-particle or field theoretic chaotic scattering S-matrix, on the modular $\operatorname{AdS}_{2}[N]$ geometry and also investigate in detail how the ETH works in this case as well as to study the corresponding scrambling time.

Finally, describing the geometry with finite dimensional, $N=p^{n}$, Hilbert multi-qudit spaces, we provided a frame- work of contact with the complexity theory of quantum algorithms and quantum circuits for the AdS/CFT correspondence [75], since the finite unitary scattering matrix can be written, as we shall show in future work, as a tensor product of elementary qudit gates.

Acknowledgements EGF and SN acknowledge the warm hospitality of the CERN Theory Division and in particular the organizers of the 2016 CERN Winter School for stimulating exchanges. SN acknowledges the warm hospitality of the Institute of Nuclear and Particle Physics of the NCSR "Demokritos". The research of EGF was partly implemented under the "ARISTEIA-I" action (Code no. 1612, D.654) and title "Holographic Hydrodynamics" of the "operational programme education and lifelong learning" and is co-funded by the European Social Fund (ESF) and National Resources. The research of MA was supported in part by the Grant MIS-448332-ORASY(NSRF 2007-13 ACTION, KRIPIS) of the European Regional Development Fund.

Open Access This article is distributed under the terms of the Creative Commons Attribution 4.0 International License (http://creativecomm ons.org/licenses/by/4.0/), which permits unrestricted use, distribution, and reproduction in any medium, provided you give appropriate credit to the original author(s) and the source, provide a link to the Creative Commons license, and indicate if changes were made.

Funded by $\mathrm{SCOAP}^{3}$.

\section{A The Weyl representation of $\operatorname{PSL}_{2}[p]$ and the construction of the QACM eigenstates}

Detailed references to this and the following appendix can be found in Refs. [28-31] and [76].

The finite Heisenberg-Weyl group, $H W_{p}$, is defined as the set of $3 \times 3$ matrices of the form

$g(r, s, t)=\left(\begin{array}{lll}1 & 0 & 0 \\ r & 1 & 0 \\ t & s & 1\end{array}\right)$

where $r, s, t$ belong to $\mathbb{Z}_{p}$ (integers modulo $p$ ), where the multiplication of two elements is carried out modulo $p$.

When $p$ is a prime integer there is a unique $p$-dimensional unitary irreducible and faithful representation of this group, given by the following matrices:

$J_{r, s, t}=\omega^{t} P^{r} Q^{s}$

where $\omega=e^{2 \pi i / p}$, i.e. the $p$ th primitive root of unity and the matrices $P, Q$ are defined as

$P_{k l}=\delta_{k-1, l}$,

$Q_{k l}=\omega^{k} \delta_{k l}$,

where $k, l=0, \ldots, p-1$.

It is to be observed that, if $\omega$ is replaced with $\omega^{k}$, for $k=1,2, \ldots, p-1$ all the relations above remain intact. Since $p$ is prime, all the resulting representations are $p$-dimensional and inequivalent. 
The matrices $P, Q$ satisfy the fundamental Heisenberg commutation relation of Quantum Mechanics in an exponentiated form,

$Q P=\omega P Q$.

In the above, $Q$ represents the position operator on the circle $\mathbb{Z}_{p}$ of the $p$ roots of unity and $P$ the corresponding momentum operator. These two operators are related by the diagonalizing unitary matrix $F$ of $P$,

$Q F=F P$,

so $F$ is the celebrated discrete Fourier transform matrix,

$F_{k l}=\frac{1}{\sqrt{p}} \omega^{k l}$, with $k, l=0, \ldots, p-1$.

An important subset of $H W_{p}$ consists of the magnetic translations

$J_{r, s}=\omega^{r s / 2} P^{r} Q^{s}$

with $r, s=0, \ldots, p-1$. These matrices are unitary $\left(J_{r, s}^{\dagger}=\right.$ $\left.J_{-r,-s}\right)$ and traceless, and they form a basis for the Lie algebra of $S L(p, \mathbb{C})$. They satisfy the important relation

$J_{r, s} J_{r^{\prime}, s^{\prime}}=\omega^{\left(r^{\prime} s-r s^{\prime}\right) / 2} J_{r+r^{\prime}, s+s^{\prime}}$.

This relation implies that the magnetic translations form a projective representation of the translation group $\mathbb{Z}_{p} \times \mathbb{Z}_{p}$. The factor of $1 / 2$ in the exponent of (A.8) must be taken modulo $p$.

The $S L_{2}(p)$ appears here as the automorphism group of magnetic translations and this defines the Weyl metaplectic representation. If we consider the action of an element

$A=\left(\begin{array}{ll}a & b \\ c & d\end{array}\right)$

on the coordinates $(r, s)$ of the periodic torus $\mathbb{Z}_{p} \times \mathbb{Z}_{p}$, this induces a unitary automorphism $U(\mathrm{~A})$ on the magnetic translations, since the representation of Heisenberg group is unitary and irreducible,

$U(\mathrm{~A}) J_{r, s} U^{\dagger}(\mathrm{A})=J_{r^{\prime}, s^{\prime}}$

where $\left(r^{\prime}, s^{\prime}\right)$ are given by

$\left(r^{\prime}, s^{\prime}\right)=(r, s)\left(\begin{array}{ll}a & b \\ c & d\end{array}\right)$.

This relation determines $U(\mathrm{~A})$ up to a phase and in the case of $A \in \mathrm{SL}_{2}[p]$, the phase can be fixed to give an exact (and not projective) unitary representation of $\mathrm{SL}_{2}[p]$.
The detailed formula of $U(\mathrm{~A})$ has been given by Balian and Itzykson [77]. Depending on the specific values of the $a, b, c, d$ parameters of the matrix $\mathrm{A}$, we distinguish the following cases:

$$
\begin{array}{ll}
\delta \neq 0: & U(\mathrm{~A})=\frac{\sigma(1) \sigma(\delta)}{p} \sum_{r, s} \omega^{\frac{b r^{2}+(d-a) r s-c s^{2}}{2 \delta}} J_{r, s}, \\
\delta=0, b \neq 0: & U(\mathrm{~A})=\frac{\sigma(-2 b)}{\sqrt{p}} \sum_{s} \omega^{\frac{s^{2}}{2 b}} J_{s(a-1) / b, s}, \\
\delta=b=0, c \neq 0: & U(\mathrm{~A})=\frac{\sigma(2 c)}{\sqrt{p}} \sum_{r} \omega^{-\frac{r^{2}}{2 c}} P^{r}, \\
\delta=b=0=c=0: & U(1)=I,
\end{array}
$$

where $\delta=2-a-d$ and $\sigma(a)$ is the quadratic Gauss sum given by

$\sigma(a)=\frac{1}{\sqrt{p}} \sum_{k=0}^{p-1} \omega^{a k^{2}}=(a \mid p) \times\left\{\begin{array}{l}1 \text { for } p=4 k+1, \\ \mathrm{i} \text { for } p=4 k-1,\end{array}\right.$

while the Legendre symbol takes the values $(a \mid p)= \pm 1$ depending on whether $a$ is or is not a square modulo $p$.

It is possible to perform explicitly the above Gaussian sums noticing that

$\left(J_{r, s}\right)_{k, l}=\delta_{r, k-l} \omega^{\frac{k+l}{2} s}$

where all indices take the values $k, l, r, s=0, \ldots, p-1$. This has been done in $[42,43]$. In the case $\delta=2-a-d \neq$ $0 \bmod p$ and $c \neq 0 \bmod p$, the result is

$U(\mathrm{~A})_{k, l}=\frac{(-2 c \mid p)}{\sqrt{p}} \times\left\{\begin{array}{c}1 \\ -\mathrm{i}\end{array}\right\} \omega^{-\frac{a k^{2}-2 k l+d l^{2}}{2 c}}$.

If $c \equiv 0 \bmod p$, then we transform the matrix $A$ to one with $c \neq 0 \bmod p$. The cases $\delta \equiv 0 \bmod p$ can be worked out easily using the expressions of the matrix elements of $J_{r, s}$, given in (A.2).

It is interesting to notice that redefining $\omega$ to become $\omega^{k}$ for $k=1,2, \ldots, p-1$, the matrix $U(\mathrm{~A})$ transforms to the matrix $U\left(A_{k}\right)$, where $A_{k}$ is the $2 \times 2$ matrix $A_{k}=\left(\begin{array}{cc}a & b k \\ c / k & d\end{array}\right)$, which belongs to the same conjugacy class with $A$ as long as $k$ is a quadratic residue. If $k=p-1$ we pass from the representation $U(A)$ to the complex conjugate one $U(A)^{*}$.

The Weyl representation presented above provides the interesting result that the unitary matrix corresponding to the $S L_{2}(p)$ element $a=\left(\begin{array}{cc}0 & -1 \\ 1 & 0\end{array}\right)$ is-up to a phase-the discrete finite Fourier transform (A.6),

$U(a)=(-1)^{k+1} i^{n} F$

where $n=0$ for $p=4 k+1$ and $n=1$ for $p=4 k-1$. 
The Fourier transform matrix generates a fourth order abelian group with elements

$F, F^{2}=S, F^{3}=F^{*}, F^{4}=I$.

The matrix $S$ represents the element $a^{2}=\left(\begin{array}{cc}-1 & 0 \\ 0 & -1\end{array}\right)$. Its matrix elements are

$S_{k, l} \quad=\delta_{k,-l}, \quad k, l=0, \ldots p-1$,

$U\left(a^{2}\right)_{k, l}=\mathrm{i}^{2 n} S_{k, l}=(-)^{n} \delta_{k,-l}, \quad k, l=0, \ldots p-1$.

Because the action of $S$ on $J_{r, s}$ changes the signs of $r, s$, while $\forall A \in S L_{2}(p)$ the unitary matrix $U(A)$ depends quadratically on $r, s$ in the sum (A.12), it turns out that $S$ commutes with all $U(A)$. Moreover, $S^{2}=I$ and we can construct two projectors

$P_{+}=\frac{1}{2}(I+S), P_{-}=\frac{1}{2}(I-S)$

with dimensions of their invariant subspaces $\frac{p+1}{2}$ and $\frac{p-1}{2}$, respectively. So the Weyl $p$-dimensional representation is the direct sum of two irreducible unitary representations,

$U_{+}(\mathrm{A})=U(\mathrm{~A}) P_{+}, U_{-}(\mathrm{A})=U(A) P_{-}$.

To obtain the block-diagonal form of the above matrices $U_{ \pm}(A)$, we rotate with the orthogonal matrix of the eigenvectors of $S$. This $p$-dimensional orthogonal matrix, dubbed here $O_{p}$, can be obtained in a maximally symmetric form (along the diagonal as well as along the anti-diagonal) using the eigenvectors of $S$ in the following order: In the first $(p+1) / 2$ columns we put the eigenvectors of $S$ of eigenvalue equal to 1 , and in the next $(p-1) / 2$ columns the eigenvectors of eigenvalue equal to -1 in the specific order given below:

$\left(e_{0}\right)_{k}=\delta_{k 0}$,

$\left(e_{j}^{+}\right)_{k}=\frac{1}{\sqrt{2}}\left(\delta_{k, j}+\delta_{k,-j}\right), j=1, \ldots, \frac{p-1}{2}$,

$\left(e_{j}^{-}\right)_{k}=\frac{1}{\sqrt{2}}\left(\delta_{k, j}-\delta_{k,-j}\right), j=\frac{p+1}{2}, \ldots, p$,

where $k=0, \ldots, p-1$.

Different orderings of eigenvectors may lead to different forms of the matrices $U_{ \pm}(A)$. The orthogonal matrix $O_{p}$ thus obtained has the property

$O_{p}^{2}=I$

due to its symmetric form.

The final block-diagonal form of $U_{ \pm}(\mathrm{A})$ is obtained through an $O_{p}$ rotation

$V_{ \pm}(\mathrm{A})=O_{p} U_{ \pm}(\mathrm{A}) O_{p}$
In the following using the above material we shall provide the details of the construction of the eigenstates of the QACM.

As we discussed in Sect. 3, the first step consists in diagonalizing the ACM in $\mathrm{SL}_{2}[p]$ and this can be done by an element $\mathrm{R} \in \mathrm{SL}_{2}[p]$ given by

$\mathrm{R}=\left(\begin{array}{cc}\frac{a-1}{2} & -\frac{1}{2}\left(a^{-1}+1\right) \\ 1 & a^{-1}\end{array}\right)$

where $a$ is the square root of $5 \bmod p$. The matrix elements of $U(\mathrm{~A})$ and $U(\mathrm{R})$ can be constructed explicitly, for any prime $p$, in particular for $p \equiv 3 \bmod 4$ :

$[U(\mathrm{~A})]_{k, l}=\langle l|U(\mathrm{~A})| k\rangle=\frac{-\mathrm{i}(-2 \mid p)}{\sqrt{p}} \omega^{-\frac{1}{2}\left(k^{2}-2 k l+2 l^{2}\right)}$

and

$[U(\mathrm{R})]_{k, l}=\langle l|U(\mathrm{R})| k\rangle=\frac{-\mathrm{i}(-2 \mid p)}{\sqrt{p}} \omega^{\frac{1}{4 a}\left((5-a) k^{2}-4 a k l+2 l^{2}\right)}$

where $k, l=0,1,2, \ldots, p-1$ and $\omega=\exp (2 \pi \mathrm{i} / p)$.

Plugging these expressions into Eq. (3.11) we obtain the explicit forms of the QACM eigenstates, $\left\langle k \mid \psi_{n}\right\rangle, k, n=$ $0,1,2, \ldots, p-1$ :

$\left\langle k \mid \psi_{0}\right\rangle=\frac{-\mathrm{i}(-2 \mid p)}{\sqrt{p}} \omega^{-\frac{(5-a) k^{2}}{4 a}}$

and

$\left\langle k \mid \psi_{n}\right\rangle=\frac{-\mathrm{i}(-2 \mid p)}{\sqrt{p(p-1)}} \sum_{\ell=1}^{p-1} e^{\frac{2 \pi \mathrm{i}}{p-1} n \operatorname{Ind}_{g} \ell} \omega^{-\frac{(5-a) k^{2}-4 a k \ell+2 \ell^{2}}{4 a}}$.

We can project $U(\mathrm{~A})$ and its eigenstates onto the two irreducible subspaces, of dimension $(p \pm 1) / 2$, obtaining the block-diagonal forms $V_{ \pm}(\mathrm{A})$

$V_{ \pm}(\mathrm{A})=O_{p} U(\mathrm{~A}) \frac{1}{2}(I \pm S) O_{p}$

and their corresponding eigenstates, $\left|\psi_{n}\right\rangle_{ \pm}$

$\left|\psi_{n}\right\rangle_{ \pm}=O_{p} \frac{1}{2}(I \pm S)\left|\psi_{n}\right\rangle$,

respectively. It can be checked that the $V_{ \pm}(\mathrm{A})$ have the same period as $U(\mathrm{~A})$. 
These two irreducible representations of SL2 $[p]$ are both appropriate for the torus, $\mathbb{T}^{2}$ but they are not, however, for realizing the projective action of $\mathrm{SL}_{2}[p]$ on $\mathrm{AdS}_{2}$, as discussed in Sect. 3. We must choose that one of the two which is, also, an (irreducible) representation of $\mathrm{PSL}_{2}[p]$ [78].

We can obtain irreducible representations of $\operatorname{PSL}_{2}[p]$ from irreducible representations of $\mathrm{SL}_{2}[p]$ in the following way: The elements

$a=\left(\begin{array}{cc}0 & -1 \\ 1 & 0\end{array}\right)$

and

$a^{2}=\left(\begin{array}{cc}-1 & 0 \\ 0 & -1\end{array}\right)$

of $\mathrm{SL}_{2}[p]$ have representatives

$U(\mathrm{a})=(-1)^{k+1} \mathrm{i}^{n} F$

and

$U\left(\mathrm{a}^{2}\right)=(-1)^{n} S$

where $n=0$ for $p=4 k+1$ and $n=1$ for $p=4 k-1$.

For $\operatorname{PSL}_{2}[p]$ the element $\mathrm{a}^{2}=-I$ is identified with the identity matrix, $I$. Therefore, we should choose, among the two irreducible representations of $\mathrm{SL}_{2}[p]$, of dimension $(p+$ $1) / 2$ and $(p-1) / 2$, the one for which $U\left(\mathrm{a}^{2}\right)=I$.

We can easily check that this happens for the $\frac{p+1}{2}$ dimensional representation, when $p \equiv 1 \bmod 4$, and for the $\frac{p-1}{2}$ dimensional one, when $p \equiv 3 \bmod 4$. In our construction we found it simpler to work with primes of the latter form; therefore the representation is that defined by $V_{-}(\mathrm{A})$, with eigenstates $\left|\psi_{n}\right\rangle_{-}$.

The corresponding eigenvalues, $\varepsilon_{n}$, in Eq. (3.10), have an index ranging in $n=(p+1) / 2, \ldots, p-1$.

\section{References}

1. G. 't Hooft, Diagonalizing the black hole Information retrieval process. arXiv: 1509.01695 [gr-qc]

2. G. 't Hooft, The quantum black hole as a hydrogen atom: microstates without strings attached. arXiv:1605.05119 [gr-qc]

3. G. 't Hooft, Black hole unitarity and antipodal entanglement. arXiv: 1601.03447 [gr-qc]

4. A. Strominger, A. Zhiboedov, Gravitational memory, BMS supertranslations and soft theorems. JHEP 1601, 086 (2016). https://doi. org/10.1007/JHEP01(2016)086. arXiv:1411.5745 [hep-th]

5. S.W. Hawking, M.J. Perry, A. Strominger, Soft hair on black holes. Phys. Rev. Lett. 116(23), 231301 (2016). https://doi.org/10.1103/ PhysRevLett.116.231301. arXiv:1601.00921 [hep-th]

6. J. Ellis, N.E. Mavromatos, D.V. Nanopoulos, $W_{\infty}$ Algebras, Hawking radiation and information retention by stringy black holes. Phys. Rev. D 94(2), 025007 (2016). https://doi.org/10.1103/ PhysRevD.94.025007. arXiv:1605.01653 [hep-th]

7. A. Strominger, Black hole information revisited. arXiv: 1706.07143 [hep-th]

8. D. Carney, L. Chaurette, D. Neuenfeld, G.W. Semenoff, Infrared quantum information. arXiv:1706.03782 [hep-th]

9. C. Barrabès, V. Frolov, R. Parentani, Stochastically fluctuating black hole geometry, Hawking radiation and the trans-Planckian problem. Phys. Rev. D 62, 044020 (2000). https://doi.org/10.1103/ PhysRevD.62.044020. arXiv:gr-qc/0001102

10. S. Banerjee, J.W. Bryan, K. Papadodimas, S. Raju, A toy model of black hole complementarity. JHEP 1605, 004 (2016). https://doi. org/10.1007/JHEP05(2016)004. arXiv:1603.02812 [hep-th]

11. K. Papadodimas, S. Raju, An infalling observer in AdS/CFT. JHEP 1310, 212 (2013). arXiv:1211.6767 [hep-th]

12. D. Stanford, L. Susskind, Complexity and shock wave geometries. Phys. Rev. D 90(12), 126007 (2014). https://doi.org/10.1103/ PhysRevD.90.126007. arXiv:1406.2678 [hep-th]

13. S.H. Shenker, D. Stanford, Multiple shocks. JHEP 1412 , 046 (2014). https://doi.org/10.1007/JHEP12(2014)046. arXiv:1312.3296 [hep-th]

14. S.H. Shenker, D. Stanford, Black holes and the butterfly effect. JHEP 1403, 067 (2014). https://doi.org/10.1007/ JHEP03(2014)067. arXiv:1306.0622 [hep-th]

15. J. Maldacena, S.H. Shenker, D. Stanford, A bound on chaos. JHEP 1608, 106 (2016). arXiv:1503.01409 [hep-th]

16. A.L. Fitzpatrick, J. Kaplan, A quantum correction to chaos. JHEP 1605, 070 (2016). arXiv:1601.06164 [hep-th]

17. P. Caputa, J. Simn, A. Štikonas, T. Takayanagi, K. Watanabe, Scrambling time from local perturbations of the eternal BTZ black hole. JHEP 1508, 011 (2015). https://doi.org/10.1007/ JHEP08(2015)011. arXiv:1503.08161 [hep-th]

18. P. Padmanabhan, S.J. Rey, D. Teixeira, D. Trancanelli, Supersymmetric many-body systems from partial symmetries integrability, localization and scrambling. JHEP 1705, 136 (2017). https://doi. org/10.1007/JHEP05(2017)136. arXiv:1702.02091 [hep-th]

19. M. Mezei, D. Stanford, On entanglement spreading in chaotic systems. arXiv:1608.05101 [hep-th]

20. J. Polchinski, Chaos in the black hole S-matrix. arXiv:1505.08108 [hep-th]

21. J. Polchinski, The black hole information problem. arXiv:1609.04036 [hep-th]

22. A.R. Brown, L. Susskind, Y. Zhao, Quantum complexity and negative curvature. arXiv:1608.02612 [hep-th]

23. J. Engelsy, T.G. Mertens, H. Verlinde, An investigation of $\mathrm{AdS}_{2}$ backreaction and holography. JHEP 1607, 139 (2016). arXiv:1606.03438 [hep-th]

24. T. Banks, W. Fischler, I.R. Klebanov, L. Susskind, Schwarzschild black holes from matrix theory. Phys. Rev. Lett. 80, 226 (1998). https://doi.org/10.1103/PhysRevLett.80.226. arXiv:hep-th/9709091

25. N. Iizuka, T. Okuda, J. Polchinski, Matrix models for the black hole information paradox. JHEP 1002, 073 (2010). https://doi.org/ 10.1007/JHEP02(2010)073. arXiv:0808.0530 [hep-th]

26. S.G. Avery, Qubit models of black hole evaporation. JHEP 1301, 176 (2013). https://doi.org/10.1007/JHEP01(2013)176. arXiv:1109.2911 [hep-th]

27. J.M. Magan, Black holes as random particles: entanglement dynamics in infinite range and matrix models. arXiv:1601.04663 [hep-th]

28. M. Axenides, E.G. Floratos, S. Nicolis, Modular discretization of the $\mathrm{AdS}_{2} / \mathrm{CFT}_{1}$ holography. JHEP 1402, 109 (2014). arXiv: 1306.5670 [hep-th]

29. M. Axenides, E. Floratos, S. Nicolis, Chaotic information processing by extremal black holes. Int. J. Mod. Phys. D 24(09), 
1542012 (2015). https://doi.org/10.1142/S0218271815420122. arXiv:1504.00483 [hep-th]

30. E. Floratos, The chaotic eigenstate hypothesis and fast scrambling on BH horizons: a quantum Arnold cat map toy model. 8th Crete regional meeting on string theory, Nafplion 2015. http://hep. physics.uoc.gr/mideast8/talks/tuesday/Floratos.pdf

31. E. Floratos, Quantum complexity and chaotic dynamics on extremal black hole horizons. 9th Crete regional meeting on string theory. http://hep.physics.uoc.gr/mideast $9 /$ talks/tuesday/floratos. pdf

32. P. Claus, M. Derix, R. Kallosh, J. Kumar, P.K. Townsend, A. Van Proeyen, Black holes and superconformal mechanics. Phys. Rev. Lett. 81, 4553 (1998). arXiv:hep-th/9804177

33. V.I. Arnol'd, A. Avez, Ergodic Problems in Classical Mechanics (Benjamin, New York, 1968)

34. G.M. Zaslavsky, Hamiltonian Chaos and Fractional Dynamics (Oxford University Press, Oxford, 2008)

35. Ya G. Sinai, Topics in Ergodic Theory (Princeton University Press, Princeton, 1994)

36. M.V. Berry, N.L. Balazs, M. Tabor, A. Voros, Quantum maps. Ann. Phys. 122, 26 (1979)

37. J.H. Hannay, M.V. Berry, Quantization of linear maps on a torus. Physica D 1, 267 (1980)

38. J. Ford, G. Mantica, G.H. Ristow, The Arnol'd cat: failure of the correspondence principle. Physica D 50, 493 (1991)

39. M.V. Berry, "Some Quantum to Classical Asymptotics", Les Houches Summer School 1989 (Elsevier, Amsterdam, 1991)

40. S. Knabe, On the quantization of Arnol'd's cat. J. Phys. A Math. Gen. 23, 2013 (1990)

41. E.G. Floratos, The Heisenberg-Weyl group on the $\mathbb{Z}_{n} \times \mathbb{Z}_{n}$ discretized torus membrane. Phys. Lett. B 228, 335 (1989)

42. G.G. Athanasiu, E.G. Floratos, Coherent states in finite quantum mechanics. Nucl. Phys. B 425, 343 (1994)

43. G.G. Athanasiu, E.G. Floratos, S. Nicolis, Fast quantum maps. J. Phys. A 31, L655 (1998). arXiv:math-ph/9805012

44. D. Bressoud, S. Wagon, Computational number theory (Key college publishing, 2000)

45. N. Koblitz, A Course in Number Theory and Cryptography (Springer, New York, 1994)

46. V.I. Arnol'd, Dynamics, Statistics and Projective Geometry of Galois Fields (Cambridge University Press, Cambridge, 2011)

47. M.V. Berry, Regular and irregular semiclassical wave functions. J. Phys. A Math. Gen. 10, 2083-91 (1977)

48. J.M. Deutsch, Quantum statistical mechanics in a closed system. Phys. Rev. A 43, 2046 (1991)

49. M. Srednicki, Quantum chaos and statistical mechanics. Ann. NY Acad. Sci. 755, 757 (1995). https://doi.org/10.1111/j.1749-6632. 1995.tb39017.x. arXiv:cond-mat/9406056

50. J.M. Magan, Random free fermions: An analytical example of eigenstate thermalization. Phys. Rev. Lett. 116, 030401 (2016). arXiv:1508.05339v2 [quant-ph]

51. B. Craps, O. Evnin, K. Nguyen, Matrix thermalization. JHEP 1702, 041 (2017). arXiv:1610.05333 [hep-th]

52. R. Hübener, Y. Sekino, J. Eisert, Equilibration in low-dimensional quantum matrix models. JHEP 1504, 166 (2015). https://doi.org/ 10.1007/JHEP04(2015)166. arXiv:1403.1392 [quant-ph]

53. J. Sonner, M. Vielma, Eigenstate thermalization in the SachdevYe-Kitaev model. arXiv:1707.08013 [hep-th]

54. D.C. Brody, D.W. Hook, L.P. Hughston, Unitarity, ergodicity, and quantum thermodynamics. J. Phys. A 40, F503 (2007). https://doi. org/10.1088/1751-8113/40/26/F01. arXiv:quant-ph/0702009

55. L. Susskind, E. Witten, The holographic bound in anti-de sitter space. arXiv:hep-th/9805114

56. M.D. Esposti, S. Isola, Distribution of closed orbits for linear automorphisms of tori. Nonlinearity $\mathbf{8 ,} 821$ (1995)
57. P. Kurlberg, Z. Rudnick, On quantum ergodicity for linear maps of the torus. Commun. Math. Phys. 222, 201 (2001). arXiv:math/9910145v1 [math.NT]

58. P. Kurlberg, Z. Rudnick, On the distribution of matrix elements for the quantum cat map. Ann. Math. 161, 489 (2005). arXiv:math/0302277v3 [math.NT]

59. F. Faure, S. Nonnenmacher, S. De Bievre, Scarred eigenstates for quantum cat maps of minimal periods. Commun. Math. Phys. 239, 449-492 (2003). arXiv:nlin/0207060v2 [nlin.CD]

60. S. Zelditch, Recent developments in mathematical quantum chaos. Curr. Dev. Math. 115-202 (2009). arXiv:0911.4312v1 [math.AP]

61. A. Sen, State operator correspondence and entanglement in $A d S_{2} / C F T_{1}$. Entropy 13, 1305 (2011). https://doi.org/10.3390/ e13071305. arXiv:1101.4254 [hep-th]

62. A. Dabholkar, S. Nampuri, Quantum black holes. Lect. Notes Phys. 851, 165 (2012). https://doi.org/10.1007/978-3-642-25947-0_5. arXiv:1208.4814 [hep-th]

63. T. Azeyanagi, T. Nishioka, T. Takayanagi, Near extremal black hole entropy as entanglement entropy via AdS(2)/CFT(1). Phys. Rev. D 77, 064005 (2008). https://doi.org/10.1103/PhysRevD.77.064005. arXiv:0710.2956 [hep-th]

64. M. Dodelson, E. Silverstein, Longitudinal nonlocality in the string S-matrix. arXiv:1504.05537 [hep-th]

65. L. Susskind, J. Lindesay, An Introduction to Black Holes, Information and the String Theory Revolution: The Holographic Universe (World Scientific, Hackensack, 2005), p. 183

66. F.J. Dyson, H. Falk, Period of a discrete cat mapping. Am. Math. Monthly 99, 603 (1992)

67. P. Hayden, J. Preskill, Black holes as mirrors: quantum information in random subsystems. JHEP 0709, 120 (2007). https://doi.org/10. 1088/1126-6708/2007/09/120. arXiv:0708.4025 [hep-th]

68. Y. Sekino, L. Susskind, Fast scramblers. JHEP 0810, 065 (2008). $\quad$ https://doi.org/10.1088/1126-6708/2008/10/065. arXiv:0808.2096 [hep-th]

69. J.L.F. Barbon, J.M. Magan, Fast scramblers and ultrametric black hole horizons. J. High Energy Phys. 2013, 163 (2013). arXiv:1306.3873v2 [hep-th]

70. S. Pramodh, V. Sahakian, From black hole to qubits: evidence of fast scrambling in BMN theory. JHEP 1507, 067 (2015). arXiv:1412.2396 [hep-th]

71. N. Lashkari, D. Stanford, M. Hastings, T. Osborne, P. Hayden, Towards the fast scrambling conjecture. JHEP 1304, 022 (2013). https://doi.org/10.1007/JHEP04(2013)022. arXiv:1111.6580 [hep-th]

72. J.L.F. Barbon, J.M. Magan, Fast scramblers of small size. JHEP 1110, 035 (2011). arXiv:1106.4786 [hep-th]

73. E. Artin, Algèbre géométrique. in ed. by J. Gabay (1996)

74. P. Shor, Polynomial-Time algorithms for prime factorization and discrete logarithms on a quantum computer. SIAM J. Sci. Stat. Comput. 26, 1484 (1997). arXiv:quant-ph/9508027

75. F. Pastawski, B. Yoshida, D. Harlow, J. Preskill, Holographic quantum error-correcting codes: toy models for the bulk/boundary correspondence. JHEP 1506, 149 (2015). https://doi.org/10.1007/ JHEP06(2015)149. arXiv:1503.06237 [hep-th]

76. E.G. Floratos, G.K. Leontaris, Discrete flavour symmetries from the Heisenberg group. Phys. Lett. B 755, 155 (2016). https://doi. org/10.1016/j.physletb.2016.02.007. arXiv:1511.01875 [hep-th]

77. R. Balian, C. Itzykson, Observations on finite quantum mechanics. C. R. Acad. Sci. Paris 303(I), 773 (1986)

78. J. Rawnsley, On the universal covering group of the real symplectic group. J. Geom. Phys. 62(10), 2044-2058 (2012). https://doi.org/ 10.1016/j.geomphys.2012.05.009. ISSN 0393-0440 